\title{
Utilizing Neurons for Digital Logic Circuits: A Molecular Communications Analysis
}

\author{
Geoflly L. Adonias, Anastasia Yastrebova, Michael Taynnan Barros, Yevgeni Koucheryavy Senior Member, IEEE, \\ Frances Cleary, and Sasitharan Balasubramaniam, Senior Member, IEEE
}

\begin{abstract}
With the advancement of synthetic biology, several new tools have been conceptualized over the years as alternative treatments for current medical procedures. As part of this work, we investigate how synthetically engineered neurons can operate as digital logic gates that can be used towards biocomputing inside the brain and its impact on epileptic seizurelike behaviour. We quantify the accuracy of logic gates under high firing rates amid a network of neurons and by how much it can smooth out uncontrolled neuronal firings. To test the efficacy of our method, simulations composed of computational models of neurons connected in a structure that represents a logic gate are performed. Our simulations demonstrate the accuracy of performing the correct logic operation, and how specific properties such as the firing rate can play an important role in the accuracy. As part of the analysis, the mean squared error is used to quantify the quality of our proposed model and predict the accurate operation of a gate based on different sampling frequencies. As an application, the logic gates were used to smooth out epileptic seizure-like activity in a biological neuronal network, where the results demonstrated the effectiveness of reducing its mean firing rate. Our proposed system has the potential to be used in future approaches to treating neurological conditions in the brain.
\end{abstract}

Index Terms-Logic gates, synthetic biology, nano communications, nanonetworks, Boolean algebra.

\section{INTRODUCTION}

It has been over a decade since Molecular Communications (MC) was introduced as a new communication paradigm aiming to conceptualize and build communication systems inspired by natural biological processes [1]-[4]. One of those MC systems is known as neuro-spike communication [5], where information is transferred between two neurons through an electro-chemical process which triggers an electrical impulse called action potentials. We are interested in the interchangeable action potentials information that comprises of

G. L. Adonias, M. T. Barros, F. Cleary and S. Balasubramaniam are with the Telecommunications Software \& Systems Group, Waterford Institute of Technology, Waterford, Ireland, e-mails: \{gadonias, fcleary, sasib\}@tssg.org.

M. T. Barros is also with the CBIG/BioMediTech in the Faculty of Medicine and Health Technology, Tampere University, Tampere, Finland. email: michael.barros@tuni.fi

A. Yastrebova and Y. Koucheryavy are with the Faculty of Information Technology and Communication Sciences, Tampere University, Tampere, Finland, e-mails: anastasia.yastrebova@tuni.fi, yk@cs.tuni.fi.

This publication has emanated from research conducted with the financial support of Science Foundation Ireland (SFI) and is co-funded under the European Regional Development Fund under Grant Number 13/RC/2077. M. T. Barros is funded by the European Union's Horizon 2020 research and innovation programme under the Marie Skłodowska-Curie grant agreement No 839553 .

Manuscript received August 30, 2019; revised October 23, 2019; accepted February 16, 2020.

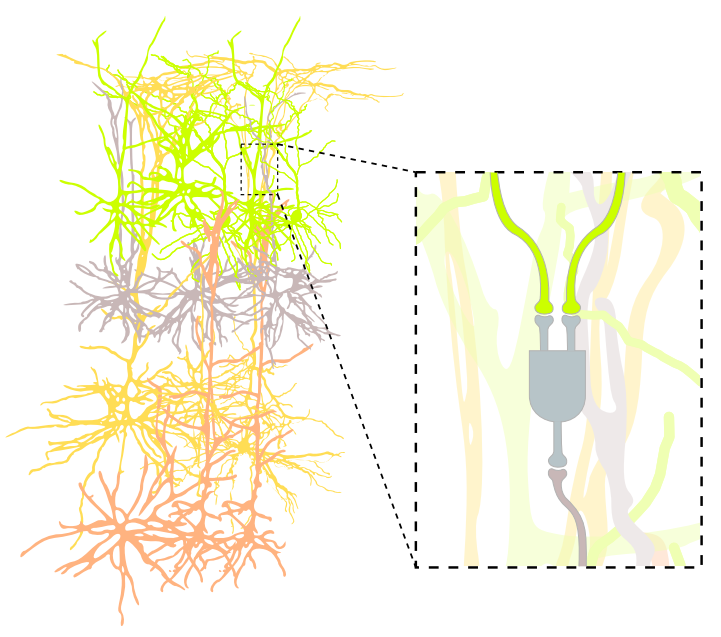

Fig. 1. Neuronal logic gate inside a cortical column.

periods with high membrane polarization, i.e. spikes, which is more suitable to analyse neural activity in population settings. This information can not only be encoded using any of the already proposed encoding techniques [6], [7], but also be modulated [8]-[10] mimicking a traditional communication system, and potentially presenting itself as a tool for cognitive enhancement and treatment of neurodegenerative diseases [11], [12].

In the 1940s, McCulloch and Pitts envisioned that the brain would be composed of units with logic gating capabilities [13]. During that time, the interest in their work in neuroscience was poor simply because neuronal cells are much more dynamic than simple digital logic gates, and there were no tools available to control such biological activity. However, since published, their seminal work contributed to advancements in artificial neural network and machine learning theories [14]. Recently, the interest in creating biological logic gates for the brain is picking up due to the emergence of cellular reprogramming towards augmenting their functioning through synthetic biology [15]. The vision of creating logic operating engineered systems to interact between natural cells and engineered cells (i.e. bio-nano machines) has the potential to create better alternatives for the treatment of diseases at the cellular level by allowing the control of their dynamics [16].

The idea of using cells in the brain as computing agents has been recently progressed by many works. One example is the work of Vogels and Abbott [17], where they investigated the signal propagation in networks of integrate-and-fire 
models of neurons and found that by either strengthening or weakening specific synapses, different types of logic gates may arise within the network. Goldental et al. [14], used neurons function and communication dynamics to propose dynamic logic gates that work based on their historical activities, interconnection profiles, as well as the frequency of stimulation at the input terminals. Song et al [18] took a different approach, where they proposed that the interaction of astrocytes in a tripartite synapse may be able to control the logic gate performance of neurons. Although they are also working with non-neuronal cells, the neurons used are all of the same morphological and electrical types. Even with this tremendous effort, these works don't explore the full impact of logic gate plurality inside a type-rich biological neuronal network (Fig. 1). Further investigation of novel logic gate constructions are needed towards more computing reliability within the chaotic activity within these networks.

Synthetic Biology has achieved success in modifying or inheriting new functions in biological cellular systems and communications [19], [20]. For the past few years, we have seen quite a lot of progress in the manipulation and engineering of the behaviour of mammalian cells [21]. This paved the way for more sophisticated approaches with regards to neuronal and non-neuronal cells (e.g. astrocytes) that can be synthetically engineered to enable control of their dynamic behaviour and functionality, aiming at the correction of abnormalities at a cellular level. With the advance of synthetic biology and nano-scale networks [22], many components ranging from logic gates [14], [17], [23], [24] to integrated circuits such as oscillators [25] have also emerged. To date, there has not been a direct application of synthetic biological-based logic gates for neurological diseases.

In light of numerous applications that can have an impact on biological systems [26], researchers have been investigating computational modelling of neurodegenerative diseases such as Alzheimer's disease (AD) [27], Parkinson's disease (PD) [28] and Epilepsy [29]. Neurodegenerative diseases can be seen as a progressive loss of specific neuronal populations that could lead to death or a disabled life. The modelling of those degeneration processes may help substantially improve our understanding which in turn has the potential to speed up the research of new therapeutic solutions. This work is inspired by the medical challenges in Epilepsy, to deliver a new system that uses synthetic biology and molecular communications. The treatment of Epileptic seizures is truly challenging, where drugs are not effective or have horrendous side effects [30]. At the same time, deep-brain stimulation techniques are not patient-friendly and Epilepsy correcting surgery has tremendously negative effects on the lifestyle of patients [31].

Information processing in the brain involves the propagation of action potentials through countless numbers of specific neuronal networks. This enables the brain to process various types of information that can range from controlling the functions of organs within an organism to coding and storing longterm memory, as examples. The synchronous uncontrolled firing of spikes in large regions of the brain that can occur spontaneously can be related to neurological diseases, and one example is epilepsy [32]. Based on this, spiking firing filtering techniques based on synthetic logic gates using, such as, digital logic gates that can improve the control of neuron activity to normal levels. This novel system can play an important role in smoothing out uncontrolled neuronal firing and consequently reducing the effects of seizures. The practical positioning of those gates also poses an issue on the feasibility of this solution. A suitable way of achieving this efficient insertion and positioning would be by using gene therapy techniques that may be invasive [33] or noninvasive [34] with the dispatch of the synthetic circuits through the bloodstream. To the best of our knowledge, there is no work in the literature on neuronal logic gates that are applicable as potential treatments for neurological disorders.

In this paper, we present a novel theoretical system that couples neuronal logic gates in a biological network of neurons. We investigate the effects of filtering high-frequency multi-unit firing caused by Epileptic seizures by randomly distributed logic gates within a validated computational framework. Unlike the aforementioned works, this paper does not perform any fine-tuning in the network, where the gates are built with three models of neurons with different morphoelectrical characteristics between each other. In our study, the type-rich neuron environment is taken into consideration for improved integration with the existing functioning network. To help quantify the ability of processing spiking information, we developed a queueing theory model that analyses the mean squared error (MSE) as a function of the inter-spike interval (ISI) at different sampling frequencies. Our work is built on top of our previous efforts [23] which only analyzed the performance of the gates as isolated units for three different inter-spike intervals (ISI) using a constant stimulus. The main contributions of this paper are as follows:

- Neuronal logic gates are built, controlled and simulated within large neuronal networks using computational models of neurons [35]. We use three models of neuron cells to create a single synthetic logic gate capable of performing logic operations at a cellular level. Two of them act as inputs so the output cell can receive stimuli from natural synaptic connections avoiding bias towards the intensity of any external stimulation.

- Analysis of performance for the gates simulated in isolation and inside a network of neurons. We analyze the dynamic behaviour of neuronal communications that could affect the operation of the gate and consequently the network, quantified in terms of accuracy. It is expected that this analysis gives an insight into how parameters of the synaptic connection and morpho-electrical characteristics of the cell, as well as the firing rate, would affect how accurately the gates process the inputs.

- Proposal of a queuing model for the input and gating of action potentials as units of information. The advantage of a queueing-theoretical model is that complex neuronal networks can be studied as a single element representing the collective behaviour of those cells. The model is capable of predicting the accuracy of the synthetic gates, and this is validated using mean 
squared error (MSE) as a function of the inter-spike interval (ISI) at different sampling frequencies.

- We quantify the impact of randomly placed logic gates in smoothing seizure-like activity. Based on the presented model of seizure-like activity, we manipulate the neuronal ionic concentration of $K$ and $N a$ to regulate the spiking rate when the disease is triggered. We couple this model with our computational framework and evaluate the decrease in mean spiking rate when neuronal logic gates are placed inside the biological neuron network.

The remainder of this paper is organized as follows, in Section II an overview of neuronal properties and how they communicate with each other is provided. The construction of neuronal logic gates, their diverse types and the queuetheoretical analysis are discussed in Section III. Section IV, presents a mathematical framework of the role played by ionic dynamics on seizure-like events is presented. Section V contains all details regarding the simulation, network connectivity and its parameters, and the results from those simulations are presented and discussed in Section VI. Finally, in Section VII the conclusions for this work are presented.

\section{Neuron COMmunicAtion BACKGROUND}

Neuronal network communications allow the propagation of spikes through a population of neurons transferring information inside the brain. Bio-computing approaches based on the communication of neurons will rely on this propagation behaviour and its relation to the neuron properties as well as the characterization of neuronal communications. Therefore, before presenting the model of logic gates using neurons (Section III), the morpho-electrical characteristics, the columnar and laminar organization properties of neurons as well as the compartmentalized Hodgkin-Huxley model for neuronal communications will be introduced.

\section{A. Neuron Properties}

Neuronal cells can be classified in terms of their morphology, electrophysiology, projections, position in the brain and the proteins and genes they express. The models of neurons used in this work, collected from [35], are classified only based on their morphological and electrical properties (morpho-electrical characteristics) as well as which cortical layer they are from (columnar and laminar organization). The classification method used in this work is detailed below.

1) Morpho-electrical Characteristics: Well-established features in the soma of the cell and its dendritic and axonal arbours are sufficient for the classification of different morphological cells. In terms of size, cortical neurons can be categorized as small neurons $(8-16 \mu m)$ along with neurons from the hippocampus, olfactory bulb and dorsal horn. Axonal features play a major role in distinguishing inhibitory types while excitatory types can be better identified by their dendritic features [35].

Different morphological types (m-types) of cells can have diverse firing patterns. These patterns are generated in response to the injection of step currents in cortical neurons. From the 11 different electrical types (e-types) identified by a) Characterized L23 PYR neuron b) Compartments c) Equivalent RC circuit

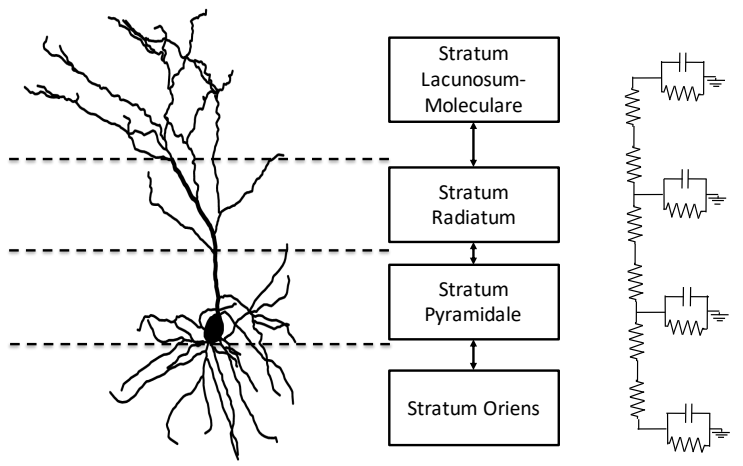

Fig. 2. Cell comparmentalization; a) A morphological structure of a layer 2/3 pyramidal neuron, b) Compartment model of a Layer 2 pyramidal neuron including 4 compartments: stratum oriens, stratum pyramidale, stratum radiatum and stratum lacunosum-moleculare, c) The equivalent RC circuit module.

Markram et al [35], all m-types used in this work are burst Non-accommodating (bNAC) e-types.

2) Columnar and Laminar Organization of the Cortex: The cerebral cortex is composed of neurons arranged into six horizontally and dispersed layers. These layers have different characteristics such as thickness, size, cell type and cell density showing a "laminar" organization and subdividing the cortex into disparate regions and areas. These layers are known as (1) Molecular layer, (2) External granular layer, (3) Pyramidal layer, (4) Inner granular layer, (5) Ganglionic layer and (6) Multiform layer.

Despite the horizontal layering, cortical regions display vertical connections that are of prime importance and take two forms: mini-columns (also called, micro-columns) with approximately $30-50 \mu \mathrm{m}$ in diameter and when activated by peripheral stimuli, it generates the macro-columns, with a diameter of approximately $0.4-0.5 \mathrm{~mm}$ [36].

\section{B. Neuronal Communications}

1) Neuron-to-neuron Communication: Communication between neurons is performed through electrochemical synapses. Action potentials travel down the axon of the pre-synaptic cell and by the time it reaches the axon terminal, it stimulates the release of synaptic vesicles inside the synaptic cleft. These vesicles contain neurotransmitters that bind to neuro-receptors in the dendrites of the postsynaptic cell, on the other end of the synaptic cleft, either depolarizing the membrane. The depolarization starts in a potential state of approximately $-65 \mathrm{mV}$ and moves up to the point it reaches a threshold which is high enough to trigger the initiation of an action potential (excitatory) or polarizing the membrane even more, which in turn blocks the postsynaptic cell of firing any spikes (inhibitory) [23], [37]. In larger networks, the balance between inhibitory and excitatory connections helps in encoding information through the neuronal network [38].

After the membrane potential reaches its maximum peak of depolarization, it starts to repolarize itself towards its resting potential right after a spike is fired. The potential gets 
hyperpolarized for a very short period which is known as refractoriness and can be subdivided into absolute and relative. During the absolute refractory period (ARP), the cell is unable to fire again regardless of how strong the stimuli are and it takes about $1-2 \mathrm{~ms}$ followed by the relative refractory period (RRP) during which a cell can fire again if the applied stimulus is stronger than it was when applied at its resting state [37].

To model such a complex system, we present a simplification of the model that is used in the NEURON simulator [39] based on the compartmentalized Hodgkin-Huxley model which is one of the most biologically plausible models for computational neuroscience [40]. We adopt the same approach as the Hodgkin-Huxley due to its mathematical tractability. In this model, the cell is broken down into $J$ equal length parts, and the spike propagation is modelled in one compartment travelling to all others (Fig. 2).

We can describe a single compartment model with the following proposed by Pospischil et al [41]

$$
C_{m} \frac{\mathrm{d} V}{\mathrm{~d} t}=-g_{\text {leak }}\left(V-E_{\text {leak }}\right)-I_{N a}-I_{K}-I_{M}-I_{T}-I_{L},
$$

where $V$ is the membrane potential, $C_{m}$ is the specific capacitance of the membrane, $g_{\text {leak }}$ is the resting (leak) membrane conductance, $E_{\text {leak }}$ is its reversal potential. $I_{N a}$ and $I_{K}$ are the sodium and potassium currents responsible for action potentials respectively, $I_{M}$ is a slow voltage-dependent potassium current responsible for spike frequency adaptation, $I_{L}$ is a high-threshold calcium current and $I_{T}$ is a lowthreshold calcium current. These voltage-dependent currents are variants of the same generic equation which is described as

$$
I_{x}=g_{x} m^{M} h^{H}\left(V-E_{x}\right),
$$

where the current $I_{x}$ is expressed as the product of the synaptic conductance, $g_{x}$, activation $(m)$ and inactivation $(h)$ variables, respectively, and the difference between membrane potential $V$ and the reversal potential $E_{x}$. Some ionic gates, such as Potassium, do not have inactivation variables so its activation is represented by the variable $n$, and described as follows

$$
I_{x}=g_{x} n^{N}\left(V-E_{x}\right)
$$

The gating of the channel is derived from the following first-order kinetic scheme

$$
C \underset{\beta(\mathrm{V})}{\stackrel{\alpha(\mathrm{V})}{\rightleftharpoons}} O,
$$

where $O$ and $C$ are the open and closed states of the gate, and $\alpha(V)$ and $\beta(V)$ are the transfer rates for each respective direction. The variables $m, n$ and $h$ represent the fraction of independent gates in the open state, following the conventional approach introduced by [42] and stated as

$$
\frac{\mathrm{d} m}{\mathrm{~d} t}=\alpha_{m}(V)(1-m)-\beta_{m}(V) m,
$$

$$
\begin{aligned}
& \frac{\mathrm{d} n}{\mathrm{~d} t}=\alpha_{n}(V)(1-n)-\beta_{n}(V) n, \\
& \frac{\mathrm{d} h}{\mathrm{~d} t}=\alpha_{h}(V)(1-h)-\beta_{h}(V) h .
\end{aligned}
$$

To consider conductance-based inputs to the neuron in (1), it is necessary to add the effects from the propagation and reception of neurotransmitters from another neuron in the synaptic cleft. We present a simplified model of the synaptic input from pre-synaptic cells, in which the neurotransmitteractivated ion channels $\left(I_{\mathrm{syn}}\right)$ is represented as an explicitly time-dependent conductance $\left(g_{\text {syn }}\right)$, and it is defined as [43]

$$
I_{\text {syn }}=g_{\text {syn }}\left(V-E_{\text {syn }}\right),
$$

where the parameter $E_{\text {syn }}$ as well as $g_{\text {syn }}$ are used to describe the many different synapses types. $E_{\text {syn }}$ may assume different values according to receptor types, the four major transmitters used for communication in the nervous systems are listed in Table I [43], where GABA means gamma-Aminobutyric acid with two different classes "A" and "B" and NMDA means N-Methyl-d-aspartic acid.

TABLE I

$E_{\text {syn }}$ FOR DIFFERENT RECEPTORS.

\begin{tabular}{c|c|c}
\hline \hline Neurotransmitter & Neuroreceptor & $E_{\text {syn }}(\mathrm{mV})$ \\
\hline Glutamate & Non-NMDA & 0 \\
Glutamate & NMDA & 0 \\
GABA & GABA $_{\mathrm{A}}$ & -70 \\
GABA & GABA $_{\mathrm{B}}$ & -100 \\
\hline \hline
\end{tabular}

Based on this, $g_{\text {syn }}$ can be defined as through a superposition of exponentials

$$
g_{\text {syn }}=\sum_{f} \bar{g}_{\text {syn }} \mathrm{e}^{-\left(t-t^{(f)}\right) / \tau} H\left(t-t^{(f)}\right),
$$

where $\tau$ is a time constant, $\bar{g}_{\text {syn }}$ is the peak synaptic conductance, $t^{(f)}$ is the arrival time of a presynaptic action potential and $H(\cdot)$ is the Heaviside step function. The $t^{(f)}$ has a nonnull value only when the membrane potential of the presynaptic compartment $V_{\text {pre }}$ crosses a threshold $t h_{\text {pre }}$, indicating a spike has occurred. This threshold-crossing mechanism for spike propagation is known as event-based synapse and it can be defined as

$$
t^{(f)}= \begin{cases}t^{(f)}, & \text { if } V_{\text {pre }} \geq t h_{\text {pre }} \\ \emptyset, & \text { otherwise. }\end{cases}
$$

This can be thought of for each synaptic event as several neurotransmitters are released and bound to the postsynaptic terminal [44].

Extending from (1) to include a new current term that comprises of the compartments that synapses may occur, we simply added the term (8) on the right-hand side, as follows

$$
C_{m} \frac{\mathrm{d} V}{\mathrm{~d} t}=-I_{l e a k}-I_{N a}-I_{K_{d}}-I_{M}-I_{T}-I_{L}-I_{\mathrm{syn}},
$$

where $I_{\text {leak }}=g_{\text {leak }}\left(V-E_{\text {leak }}\right)$. 


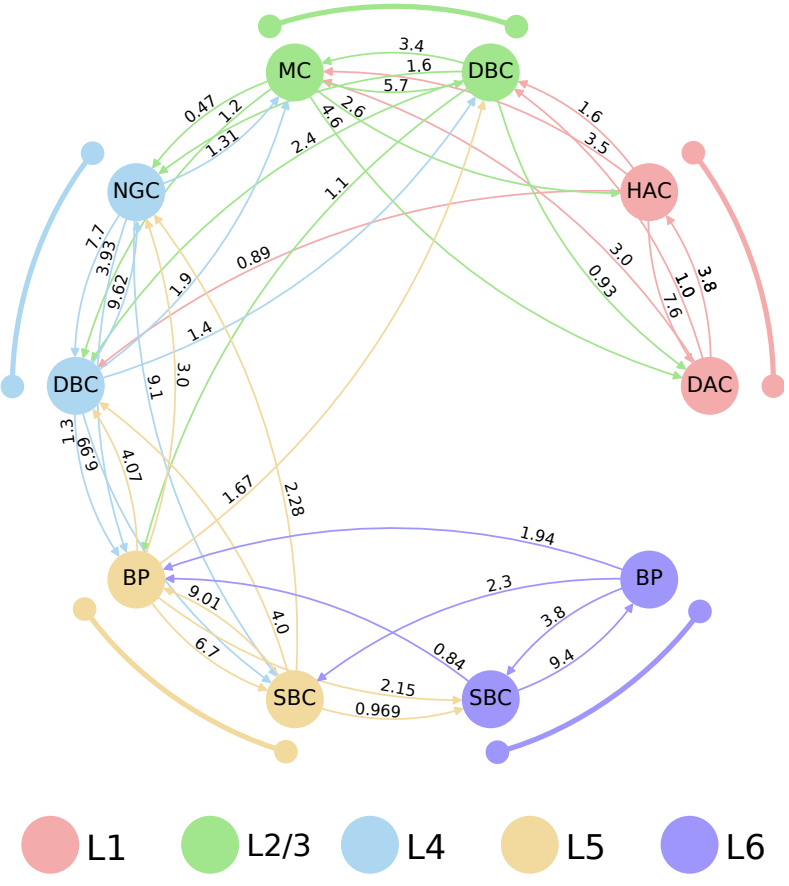

Fig. 3. Graphical visualization of network connectivity and each connection probability (in percentage) between pairs of neurons.

In Section II-A, we presented the differences of morphoelectrical characteristics of neurons and their columnar and laminar organization that create a variety of neuronal networks with different types of cells. To incorporate these properties into the model presented in this section we need to use three different approaches. Their morphological properties will dictate the number of compartments of a cell type, which probably indicates that pyramidal, granule and fusiform cells will have different $V$ propagation patterns based on their different number of compartments. For example, in Fig. 2, we divided a layer 2 pyramidal neuron into 4 compartments (stratum oriens, stratum pyramidale, stratum radiatum and stratum lacunosummoleculare). By using the NEURON simulator, we can capture morphological properties with precision through the validated models of Markram et al [35] in which compartments are already provided. In a similar manner, also using the models from [35], it is necessary to change the following parameters in order to shape the electrical characteristics and obtain a typespecific spiking activity, other than the types already available: $m, n, h, \alpha(V), \beta(V), g_{x}, g_{s y n}$, and initial values of $E_{l e a k}$ and $E_{\text {syn }}$. Lastly, based on the cell type, we define a network of excitatory neurons that consider the connection probabilities between them as defined in the Neocortical Microcircuit Collaboration Portal ${ }^{1}$. We use a simple directed graph to capture this network connectivity pattern, as shown in Fig. 3. Since the model presented in that portal went through a complete validation work, and since we use their models including the constrains of connection probabilities and synaptic weight, our model is in accordance to their computational approach and simulations.

\footnotetext{
${ }^{1}$ https://bbp.epfl.ch/nmc-portal/welcome
}

2) Role of the Threshold in Event-based Synapses: As aforementioned in Section II-B1, for a spike to be fired, the membrane potential of the cell compartment to reach a threshold during its depolarization state, the threshold for spike initiation varies with stimuli, cell type and the history of activity of the cell. It is not yet clear what characteristics can cause this variability which may affect the performance of the gate. According to Platkiewicz and Brette [45], even though the concept of spike threshold may be different for in vivo, in vitro and computational experiments, the threshold in brain cells depends on several parameters such as stimulus, type of cells, synaptic conductances and properties of ionic channels.

For a synapse, with each action potential arrival at the presynaptic terminal at time $t^{(f)}$, a specific number of neurotransmitters may be released into the synaptic cleft and has a probability of binding to the neuroreceptors at the postsynaptic cell. This release process is proportional to the shape and energy of the incoming action potential. An eventbased synapse mimics this chemical process and sends an event with a synaptic weight to the postsynaptic cell that may trigger an action potential and consequently propagate information through the network.

To the best of our knowledge, there are no works that utilize realistic models of neurons, and especially the neuron models proposed by Markram et al [35], where the gates are constructed from heterogeneous neuronal arrangements and controlled by their respective threshold for event-based synapses.

\section{Neuronal Digital Logic Gates and Circuits}

In this section, we describe the construction of neuronal logic gates and how queueing theory can be applied to neuronal circuits to predict and assess how the stimuli in the pre-synaptic terminal are being processed by the post-synaptic cell.

\section{A. Single Logic Gates}

Eight neuronal logic gates were built, including five different OR gates and three different AND gates. The truth table for both of these types of gates is depicted in Table II.

TABLE II

TRUTH TABLE FOR BOTH GATE TYPES.

\begin{tabular}{c|c|c|c}
\hline \hline \multicolumn{4}{c}{ Truth Table } \\
\hline $\mathrm{I}_{1}$ & $\mathrm{I}_{2}$ & $\mathrm{O}_{\text {AND }}$ & $\mathrm{O}_{\mathrm{OR}}$ \\
\hline 0 & 0 & 0 & 0 \\
0 & 1 & 0 & 1 \\
1 & 0 & 0 & 1 \\
1 & 1 & 1 & 1 \\
\hline \hline
\end{tabular}

For an AND gate, both inputs must be non-null to have a non-null output. On the other hand, an OR gate can send out non-null outputs not only when both inputs are active but also when either one of them is active while the other is not. All cell types used to build the gates are listed in Table III [35], [46]. 
TABLE III

TYPES OF CELLS USED TO BUILD THE GATES.

\begin{tabular}{c|c|c}
\hline \hline \multicolumn{3}{c}{ Cell Types } \\
\hline \multirow{3}{*}{ L1 } & DAC & Descending Axon Cell \\
& HAC & Horizontal Axon Cell \\
& SAC & Small Axon Cell \\
\hline \multirow{4}{*}{ L2/3 } & MC & Martinotti Cell \\
& NBC & Nest Basket Cell \\
& BTC & Bitufted Cell \\
& DBC & Double Bouquet Cell \\
& LBC & Large Basket Cell \\
\hline \multirow{3}{*}{ L4 } & DBC & Double Bouquet Cell \\
& SBC & Small Basket Cell \\
& MC & Martinotti Cell \\
\hline \multirow{2}{*}{ L5 } & BP & Bipolar Cell \\
& SBC & Small Basket Cell \\
\hline L6 & MC & Martinotti Cell \\
\hline \hline
\end{tabular}

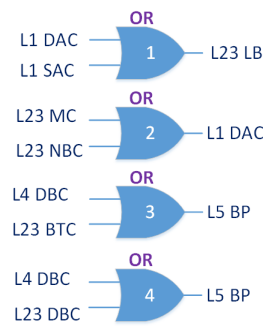

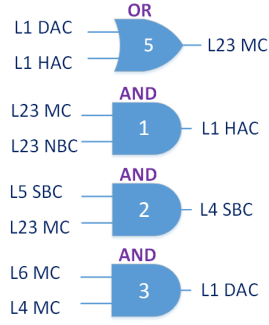

(a)

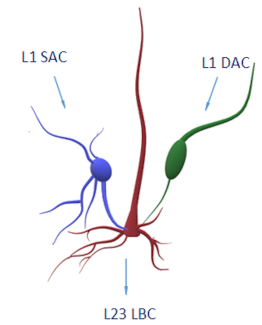

(b)
Fig. 4. Neuronal logic gates; (a) set of neuronal logic gates built using the models of neurons shown in Table III in a traditional representation and (b) potential real connection of neurons as a gate (merelly illustrative).

For each gate, three different types of cells were arranged in a way that two of them should operate as the inputs of the gate and the third one as the output (Fig. 4(b)). The idea is to keep the inner connections of the gate, i.e. the connection between the inputs cells with the output cell, fixed at their default parameters and respective connection probabilities according to the type of cells being connected.

Combinations of cells (as illustrated in Fig. 4(a)) were created largely based on their respective connection probabilities. Since the synaptic weight was kept at a fixed starting value, the higher the probability of two cells establishing a synapse, the higher the influence of the pre-synaptic cell on the postsynaptic cell. In this case, OR gates should have stronger inner connections when compared to AND gates so we can achieve the desired behaviour, as presented in Table II.

In this work, a simple On-Off Keying (OOK) modulation is implemented where a spike is considered as a bit ' 1 ' and its absence a bit ' 0 ' in each time slot (usually $5 \mathrm{~ms}$ long) for the inputs into the synthetic gates. The example spikes that propagate along each neuron of a gate is illustrated in Fig. 5. When reproducing a $[1,1]$ input with both L1-HAC and L1-DAC cells (Fig. 5 left side), the spikes should arrive at L23-MC with a minimum amount of time shift between the spikes to avoid misprocessing of the inputs by the output cell.

\section{B. Queuing Theory in Neuronal Circuits}

Queueing theory is applied in our analysis to evaluate the response time and accuracy of the proposed neuronal logic

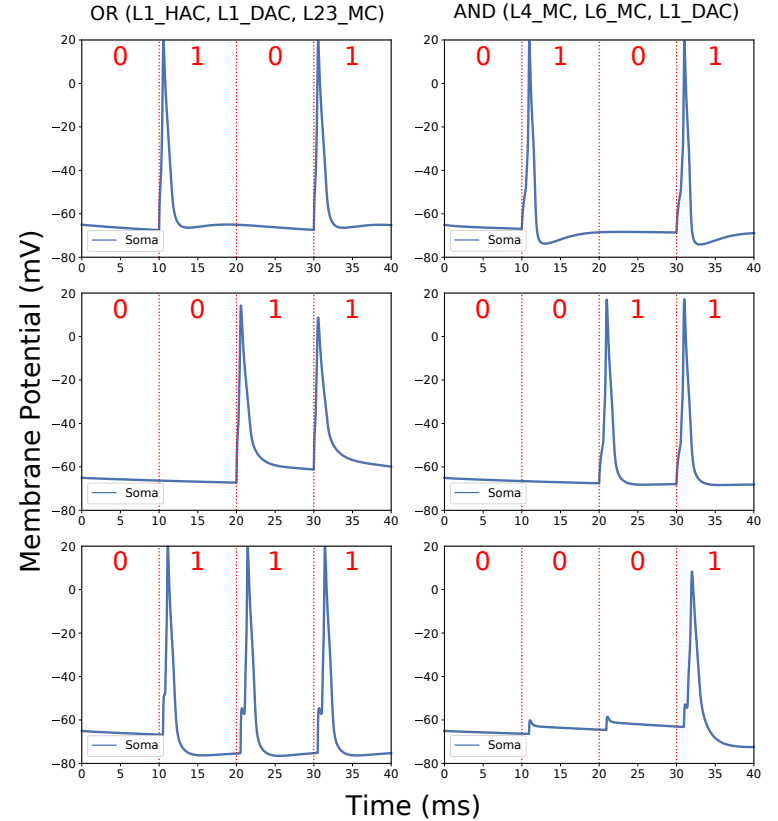

Fig. 5. Basic simulation with inputs $[0,0],[0,1],[1,0]$ and $[1,1]$ for both OR and AND gates with a 10-ms time slot. Inputs 1 and 2 are the first and second rows respectively, the last row is the output.

gates. When looking into the times of arrival of spikes, in other words, considering only the electrical behaviour of an electrochemical synapse, even though there are two inputs, we assume that there is only one queue at the server in which the inputs arrive at a unified rate. At any given moment, only one impulse is carried by the cell and any impulse coming at a rate higher than the service rate may be lost, otherwise, the cell may be able to carry the stimulus and fire again if the input is strong enough to trigger an action potential. The server utilization over a certain period, however, depends on the rate of the impulse arrival to the presynaptic terminal.

1) Queueing Analysis: Consider three neuronal cells arranged as a gate, as illustrated in Fig. 4(b), in which two of them are inputs 1 and 2, respectively, and the third cell is the output. We assume that inputs 1 and 2 have poissonic rates of $\lambda_{1}$ and $\lambda_{2}$ spikes per second, respectively, and the output cell "processes" those inputs with a rate of $\mu$ spikes per second.

Let's also consider that each input has an individual interspike interval, $\Delta I_{1}$ and $\Delta I_{2}$, and an inter-neuronal spike interval between both inputs, $\Delta I_{N}$. It is safe to assume that from the perspective of the output cell, the inputs have a merged rate, $\lambda$, defined as [47]

$$
\lambda=\lambda_{1}+\lambda_{2}
$$

which means that there is only one input with rate $\lambda$ and, analogously, $\Delta I_{N}$ as a unified inter-spike interval. In other words, inputs arrive at time $t^{(f)}+k \cdot \Delta I_{N}$, as depicted in (10), where $k$ is a zero-indexed order of arrival.

The system now looks like a single-queue and single-server (Fig. 6). However, if $\mu<\lambda$, there will be no waiting time and, any spike that is not processed on a first come first serve basis, will be lost. 


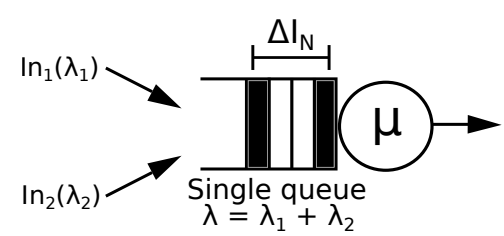

Fig. 6. Illustration of merged rates of pre-synpatic spikes into a single queue to be processed by a single server as described in Section III-B1. $\Delta I_{N}$ may have different values for OR and AND gates.

In the case of an OR gate, the output cell should fire when either of the inputs or even both of them fire, hence

$$
\Delta I_{1,2} \geq 2 \cdot \Delta I_{N}
$$

on the other hand, a cell working as an AND gate should fire only when both inputs fire together. So

$$
\Delta I_{1,2} \leq R p+\Delta I_{N}
$$

where $R p$ is the refractory period of the output cell. There are different rules for the value of $\Delta I_{N}$ when either input fires (OR gate) or both inputs fire (both gates), thus

$$
\begin{cases}\Delta I_{N} \geq R p, & \text { either input fires, } \\ 0 \leq \Delta I_{N} \leq t_{s}, & \text { both inputs fire, }\end{cases}
$$

where $t_{s}$ is some threshold in milliseconds allowing the cell to process neighbouring spikes as $[1,1]$ input.

If an input arrives with time $t^{(f)}(9)$, then the probability of another input arriving before $t^{(f)}+t_{s}$ is

$$
P\left(1 \mid\left[t^{(f)}, t^{(f)}+t_{s}\right]\right)=1-e^{-\lambda t_{s}}
$$

where for an AND gate, the smaller the $t_{s}$, the better to evoke a spike in the output. In the case of an OR gate, we are also interested in another input arriving after $t^{(f)}+t_{s}$, which will transform into

$$
P\left(1 \mid\left[t^{(f)}, t^{(f)}+t_{s}\right]\right)=e^{-\lambda t_{s}}
$$

Using both (16) and (17) and by setting a probability threshold for the reconstruction of the queue, it is possible to predict the output of the server with server rate $\mu$ which is based on the type of gate and the rate $\lambda$ and then calculate the accuracy in relation to the expected output in which both inputs are known. This accuracy should be compared to the approach for calculating the difference between the actual output of the gate and the expected output. The model is further validated in Section VI-A, where we employ the Mean Squared Error (MSE) analysis.

\section{IONIC CONCENTRATION DyNAMICS ON SEIZURE-LIKE EVENTS}

Modelling of neurodegenerative diseases is a hot topic under the area of computational pathology. Many models have been proposed with great advancements on their validation through wet-lab experiments [48]-[50]. They generally are based on different modelling approaches. However, approximations of their neural activity can be relied on conventional approaches to not particularly describe the disease but to quantify the impact of it on the general biological system functions [51].

The Hodgkin-Huxley equations, used in our modelling, make the reasonable assumption that intra- and extracellular ion concentrations of sodium and potassium are constant although it is not clear yet how valid this assumption can be for other cases. In mammalian brains, typical ionic currents may have a higher impact on ion concentrations because the neurons are small and the networks are very dense [52]. By looking at their dynamics, one can regulate the spiking rates obtained by the conventional Hodgkin-Huxley model and mimic both the normal and seizure-like neural activity.

Several types of epilepsy have been implicated with deficiencies in extracellular potassium $\left([K]_{o}\right)$ regulation. In order to take into account the effects of ion accumulation and regulation, let's first present the reversal potential $E_{x}$ (Section II-B, Equations (2) and (3)) in terms of the instantaneous intra- and extracellular ion concentrations

$$
\begin{aligned}
E_{N a} & =26.64 \cdot \ln \left(\frac{[N a]_{o}}{[N a]_{i}}\right) \\
E_{K} & =26.64 \cdot \ln \left(\frac{[K]_{o}}{[K]_{i}}\right) .
\end{aligned}
$$

where $E_{N a}$ and $E_{K}$ are the reversal potential of the Sodium and Potassium channels respectively.

The dynamics of the concentration of extracellular potassium and intracellular sodium ions are given by

$$
\begin{gathered}
\tau \frac{d[K]_{o}}{d t}=\gamma \beta I_{K}-2 \beta \tilde{I}_{\text {pump }}-\tilde{I}_{\text {glia }}-\tilde{I}_{\text {diff }}, \\
\tau \frac{[N a]_{i}}{d t}=-\gamma I_{N a}-3 \tilde{I}_{\text {pump }}
\end{gathered}
$$

where the concentrations are in $\mathrm{mM}, \tau=10^{3}$ balances the time units, $\gamma=4.45 \times 10^{-2}$ is a factor that converts the membrane currents into $\mathrm{mM} / \mathrm{s}, \beta=7$ is the ratio of intracellular to extracellular volume and $I_{K}$ and $I_{N a}$ refer to the ionic currents first described in Equations 2 and 3. The pump, glia and diffusion molar currents (also measured in $\mathrm{mM} / \mathrm{s}$ ) are given by

$$
\begin{gathered}
\tilde{I}_{\text {pump }}=\rho\left(\frac{1}{1+e^{\left(8.33-0.33[N a]_{i}\right)}}\right) \cdot\left(\frac{1}{1+e^{\left(5.5-[K]_{o}\right)}}\right) \\
\tilde{I}_{\text {glia }}=G\left(1+e^{\left(7.2-0.4[K]_{o}\right)}\right)^{-1} \\
\tilde{I}_{\text {diff }}=\varepsilon\left([K]_{o}-k_{\text {bath }}\right)
\end{gathered}
$$

where the default parameters are set as $\rho=1.25 \mathrm{mM} / \mathrm{s}$, $G=66.666 \mathrm{mM} / \mathrm{s}$, and $\varepsilon=1.333 \mathrm{~Hz}$ and $k_{\text {bath }}=4$ $\mathrm{mM}$ represents the potassium concentration in the reservoir. The intracellular potassium $\left([K]_{i}\right)$ and extracellular sodium $\left([\mathrm{Na}]_{o}\right)$ concentrations are obtained as

$$
[K]_{i}=140 \mathrm{mM}+\left(18 \mathrm{mM}-[N a]_{i}\right),
$$




$$
[N a]_{o}=144 \mathrm{mM}-\beta\left([N a]_{i}-18 \mathrm{mM}\right),
$$

where it is assumed the total amount of sodium is conserved, sodium is transported across the membrane predominantly through sodium membrane current and there is a relation between the transport of both sodium and potassium [52].

\section{Simulation Model}

In this section, the simulation model for a single neuronal logic gate as well as the application case-study scenario for the suppression of epilepsy, are presented.

\section{A. Single Gate}

The single neuronal logic gates were simulated in two ways. First, they were individually analyzed and simulated in isolation, where their respective accuracy values were evaluated and those results were fitted to the model described in Section III-B1. In isolated form, their configuration is illustrated in Fig. 4(b).

For all simulations, intrinsic parameters of the cell were kept at their default values (such as the length and diameter of each compartment of the cell), and all other parameters of the simulator required to reproduce the desired behaviour are shown in Table IV, where synaptic weight influences the spiking behaviour of one neuron has on another through either exciting or inhibiting the post-synaptic cell by setting to positive or negative values respectively; the time slot for sampling the spike train is set to $5 \mathrm{~ms}$ as a fair amount of time to account for absolute and relative refractory periods [37]; the noise object is set to 1 to mimic a Poisson firing; tau is the decay time constant of the synapse; threshold accounts for the detection of a synaptic event as described in Section II-B; delay is the time between source crossing threshold and delivery event to target; the threshold for spike detection is used to sample the spike trains into bits where any potential higher than $0 \mathrm{mV}$ in a specific time slot is a bit " 1 ". The values of $R p$ and $t_{s}$ are only used when simulating the queue model and they are not part of the simulation of the network.

TABLE IV

PARAMETERS FOR SIMULATION.

\begin{tabular}{c|c}
\hline \hline \multicolumn{2}{c}{ Simulation Parameters } \\
\hline Synaptic weight & $0.04 \mu \mathrm{S}$ \\
Simulation time & $1 \mathrm{~s}$ \\
Time slot & $5 \mathrm{~ms}$ \\
NetStim.noise & 1 \\
ExpSyn.tau & 2 \\
NetCon.threshold (AND) & $-64 \mathrm{mV}$ \\
NetCon.threshold (OR) & $5 \mathrm{mV}$ \\
NetCon.delay & 0 \\
Threshold (spike detection) & $0 \mathrm{mV}$ \\
\hline$R p$ & $5 \mathrm{~ms}$ \\
$t_{s}$ & $0 \mathrm{~ms}$ \\
\hline \hline
\end{tabular}

1) Accuracy: All gates were tested in terms of accuracy with variations in a few parameters to test their performance. These parameters include their firing rate, $\lambda$, and the synaptic threshold, th. As mentioned earlier in Section III-A, we are using an OOK modulation to discretize the spiking activity into binary code. Action potentials can shift and get slightly delayed during propagation, and this is due to axonal characteristics. This emphasizes the importance of having a time slot with a fair length of time so there is a fair distinction between different input combinations. The accuracy will measure how correct the bit train is from the output cell with regards to the ideal output that would be generated by an error-free logic gate. In the simulations, random spike trains following a Poisson process were stimulated. Poisson process usually provides good approximations of the randomness of spike trains across several trials [53]. For each simulation, since the input is random, the number of spikes fired between both inputs with the same rate is approximately the same.

The accuracy is calculated according to the following equation [54]:

$$
A(E[Y] ; Y)=\frac{P_{1,1}+P_{0,0}}{P_{1,1}+P_{1,0}+P_{0,1}+P_{0,0}}
$$

where $P_{Y, E[Y]}$ is the probability of $Y$ given $E[Y]$ in which $Y$ is the actual output and $E[Y]$ is the expected one and $Y \& E[Y] \in\{0,1\} . P_{Y, E[Y]}$ can be analogously defined as the conditional probabilities in a binary symmetric channels (BSC). Hence, $P_{0,0}=1-P_{1,0}$, and $P_{0,1}=1-P_{1,1}$. We can calculate $P_{1,1}$, for example, basically by counting the number of bits there are for each input-output combination, for example, the number of times bit 1 was sent and bit 1 was received $\left(\# B_{1,1}\right)$ and, also, bit 1 was sent and bit 0 was received $\left(\# B_{0,1}\right)$, then $P_{1,1}=\# B_{1,1} /\left(\# B_{1,1}+\# B_{0,1}\right)$.

It is expected that the threshold for a synaptic event will impact the accuracy results. As stated in Section II-B, the threshold of the cell for initiation of an action potential is dynamic and besides the synaptic events arriving at its presynaptic terminals, morphological and electrical characteristics of the cell also play a role as well as the rate with which these events arrive. This means that a small change in the way a post-synaptic neuron detects an input triggers changes in other processes that affect the depolarization of its membrane. This can lead to low accuracy results where false positive or false negative results emerge affecting the system reliability.

2) Mean Squared Error (MSE): To validate the model proposed in Section III-B, we estimated how far away from our predictions were from the values of accuracy by using Mean Squared Error. MSE is a way to measure the quality of an estimator, and in our case, we want to determine the effectiveness of our model concerning the real accuracy of the gate operation.

Consider that $A$ is the actual accuracy obtained from the real output and that $\bar{A}$ is the predicted accuracy estimated by our model, then the MSE for each point can be calculated as

$$
M S E=\frac{1}{a} \sum_{i=1}^{a}\left(A_{i}-\bar{A}_{i}\right)^{2},
$$


where $a$ is the number of accuracy values. The value of $a$ is the bandwidth of the spiking activity or the number of different frequencies with which the simulation is performed.

\section{B. Neuronal Activity Behavior During Epileptic Seizures}

Neuronal synchronization is the basis for fundamental brain processes. In neurological diseases, such as epilepsy, neuronal synchronicity, as well as the balance between excitation and inhibition in populations of cortical neurons, can be modified [55], [56].

In this work, a study on brain seizures is conducted by simulating the activity of neurons grouped in a cortical column and by reproducing the stages of spikes before, during, after, and recovery periods of epileptic seizures with a dynamic firing rate. The frequencies for stimulation varies according to results published by Alvarado-Rojas et al [55]. In a simulation with time $T=1000 \mathrm{~ms}$, the ranges of the firing rate $\gamma$ (spikes/s) for the various stages of the epileptic seizure in the network are based on the following values

$$
\gamma= \begin{cases}10-30, & \text { if } T \leq 300 \\ 10-70, & \text { if } 300<T \leq 650 \\ 70-180, & \text { if } 650<T \leq 750 \\ 0-10, & \text { if } T>750\end{cases}
$$

where the evoked activity of the network also follows a Poisson process. This procedure can also be described with the pseudo-code presented in Algorithm 1.

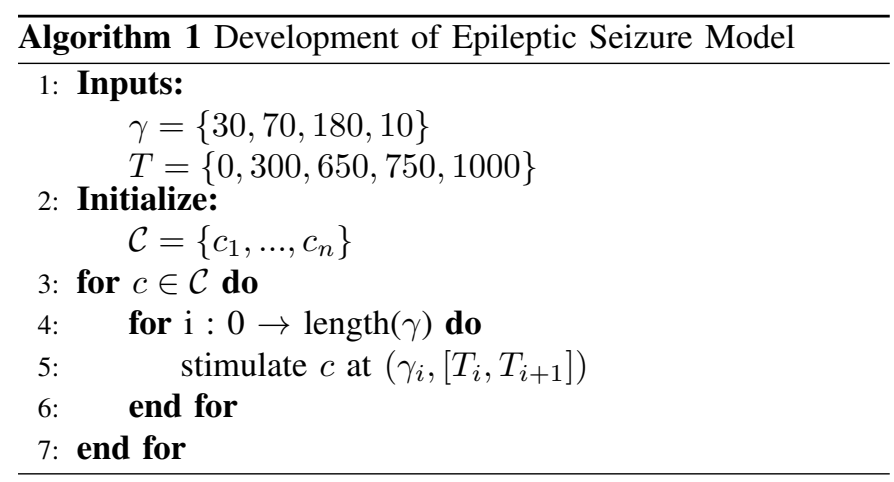

Neuronal AND logic gates were inserted inside a network with 10 neurons (two neurons per cortical layer) which was built as a simpler model of a cortical micro-column to help evaluate the effects of logic gates inside a neuronal network that simulated different stages of an epileptic seizure. The positioning of the gates between cortical layers is depicted in Fig. 7.

\section{RESULTS AND DisCUSSIONS}

In this section, we present a discussion over the results for the logic gate analysis and its epilepsy case study.

\section{A. Logic Gate Performance}

For the single gate performance accuracy, we use the configurations that are presented in Fig. 4(a). In the simulations with isolated gates, two different analyses were performed.

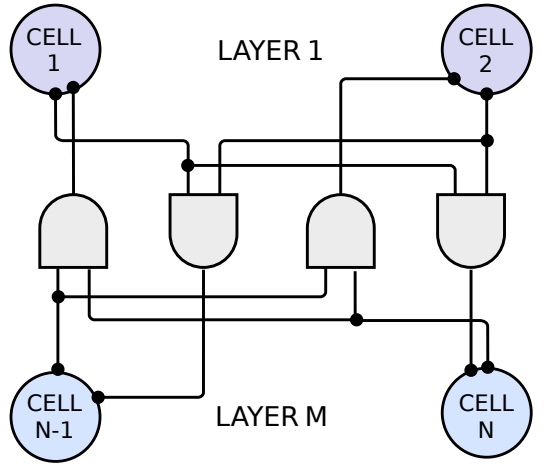

Fig. 7. Schematic of the connection of the neuronal logic gates in between the $N$ cells that forms the network with $M$ layers. In this work $N=10$ and $M=5$ where there are $N / M$ cells per layer. The placement of a logic gate in the network require the breakage of the natural connections between the cells.

First, the spiking rate was increased and the accuracy of the gates were computed, and the simulations used the parameters shown in Table IV. In both Figs. 8(a) and 8(b), the accuracy decreases as the firing rate increases, but they are decreasing at different rates due to their different behaviours as shown in Table II and Fig. 5. Even though the different versions of both types of gates have very similar values of accuracy, in Fig. 8(a) all of the AND gates configurations have very similar behaviour, this may be due to the fact that, as depicted in Table II, there is only one way for the gate to fire which decreases the chances of misprocessing the inputs. Meanwhile, since OR gates have more ways of firing an output (Table II), the different arrangements may be affecting the processing of the inputs by the output considering that the gates have a bit less similar performance between each other when compared to the performance of AND gates. Fig. 8(b) shows that OR 2 slightly stands out in performance with better accuracy compared to the other OR gates. To obtain the mean and standard deviation, the simulation ran five times.

Second, two gates were picked out, one of each type (OR2 because it showed a better accuracy in Fig. 8(b) and, AND1 was actually picked at random since all AND gates have a similar performance), and the accuracy was computed based on both the accuracy and the threshold at the presynaptic compartment. In Fig. 9, it is noticeable that the accuracy has a relationship with the threshold, $t h_{\text {pre }}$ in (10), and spiking rate of the neurons, $\lambda_{1,2}$ in (12). As previously discussed, it is expected that event-based thresholds impact the accuracy results of the logic gates. Since the threshold for initiation of an action potential of biological neurons is dynamic, and not static as some artificial models of neurons, the spiking rate is also considered as dynamic since the synaptic events arriving at a postsynaptic cell, morphological and electrical characteristics of the cell also play a role [45]. Generally speaking, lower spiking rates with higher thresholds negatively affects the accuracy of both types of gates. The results presented are based on the mean from three simulation runs.

The effect of shifting the synchronization of the spikes by up to $4 \mathrm{~ms}$ was also analyzed. The results, depicted in Table V, did not show any specific trend when the shift was 
TABLE V

ACCURACY MEAN AND STANDARD DEVIATION VALUES FOR DIFFERENT DELAYS BETWEEN INPUTS.

\begin{tabular}{c|c|c|c|c|c|c|c|c}
\hline \hline \multicolumn{1}{c}{ Effect of Delay on Accuracy } \\
\hline Types & AND 1 & AND 2 & AND 3 & OR 1 & OR 2 & OR 3 & OR 4 & OR 5 \\
\hline Mean & 0.9660 & 0.9530 & 0.9954 & 0.7595 & 0.8595 & 0.7630 & 0.7615 & 0.7720 \\
\hline Std Dev & 0.00577 & 0.00604 & 0.00603 & 0.02871 & 0.02752 & 0.03059 & 0.03405 & 0.03039 \\
\hline \hline
\end{tabular}

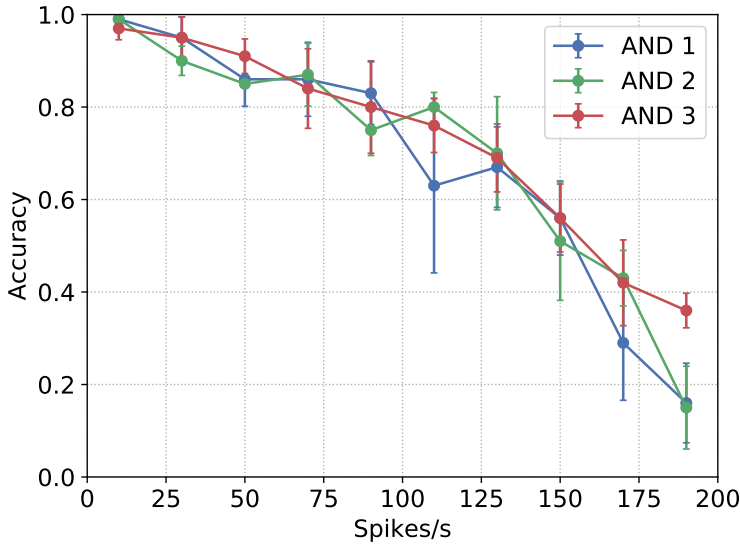

(a)

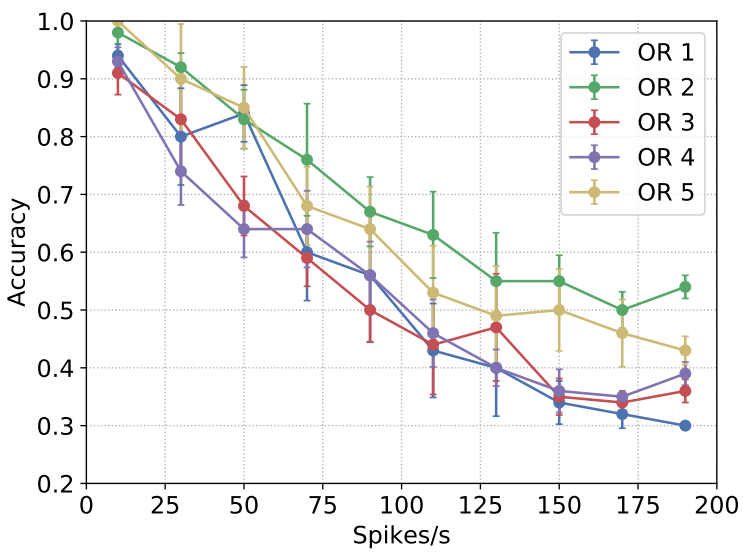

(b)

Fig. 8. Mean and standard deviation of the accuracy for the (a) three AND gates and (b) five OR gates. Five simulations were performed for each rate and the firing of the spikes follows a Poisson process.

increased. For the AND gates, the accuracy remained above $95 \%$, which represent a difference of at least $9 \%$ concerning OR gates where the highest performance is approximately $86 \%$. The highest standard deviation for an AND gate is still over 50 times smaller than the highest value for an OR gate, as aforementioned, this may be due to their different gating behaviours.

The prediction model was analyzed in relation to $\Delta I_{N}$ and its performance is presented for two gates AND1 (Fig. 10(a)) and $O R 2$ (Fig. 10(b)). These accuracy values were calculated with a five-millisecond time slot for the discretization analysis of the output cells. The other performances were omitted to avoid redundancy but MSE values are presented in Fig. 11 for all of the eight built gates. The results presented in Fig. 10

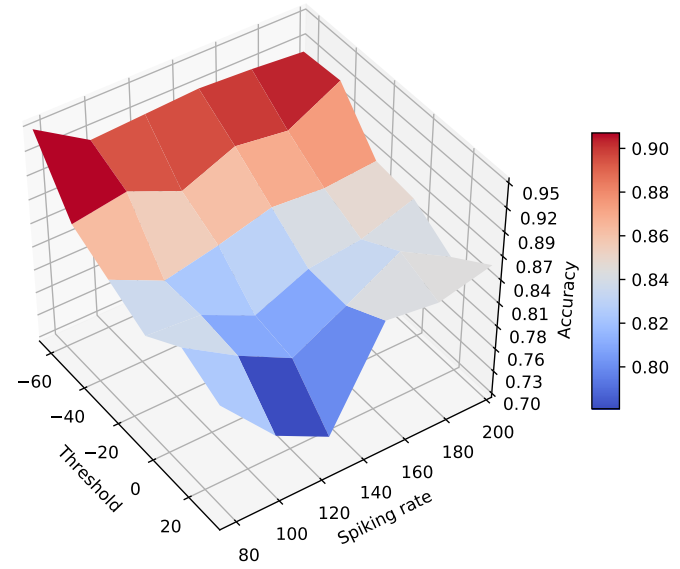

(a)

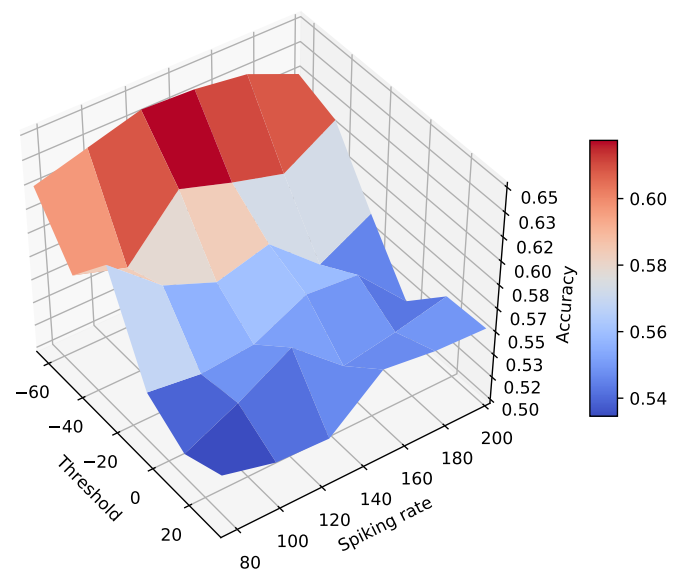

(b)

Fig. 9. Spiking rate $\lambda$, threshold $t h$ and mean accuracy $A c c$ for gates (a) AND1 and (b) OR2.

shows that for the AND gates, the model results in slightly higher accuracy compared to the OR gate. This difference between types of gates reveals itself for the other six gates, four of the OR type and two of the AND type.

In Fig. 11, the sampling frequency was changed to evaluate how the shift among bits from both inputs affect the results of our model in comparison with the real firing of the gates.

Figs. 11(a)-11(c) shows the MSE for AND gates with $\Delta I_{N}$ equal to 1,3 , and $5 \mathrm{~ms}$ respectively. On the other hand, Figs. 11(d)-11(f) show results for the same analysis but OR gates. In both scenarios, the best MSE was for a time slot with a length of $1 \mathrm{~ms}$, even though an action potential takes a longer period than the firing as well as the duration to pass the absolute refractory period. For all gate models, the MSE 


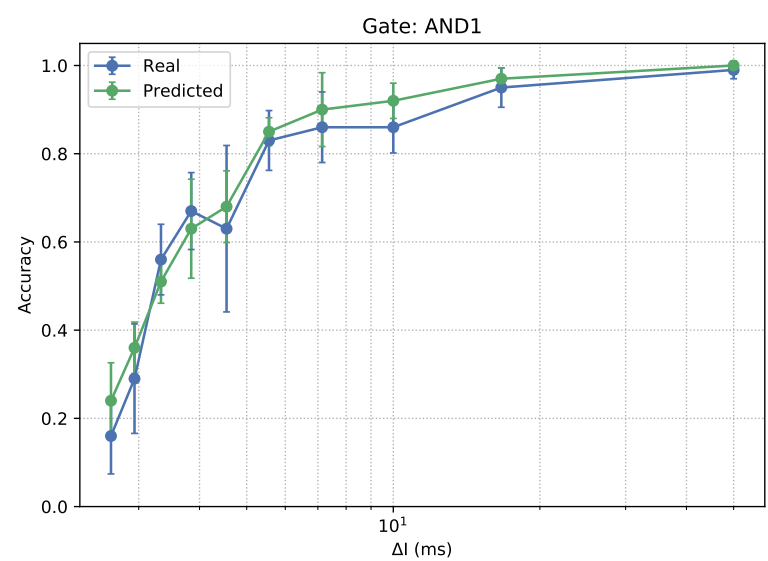

(a)

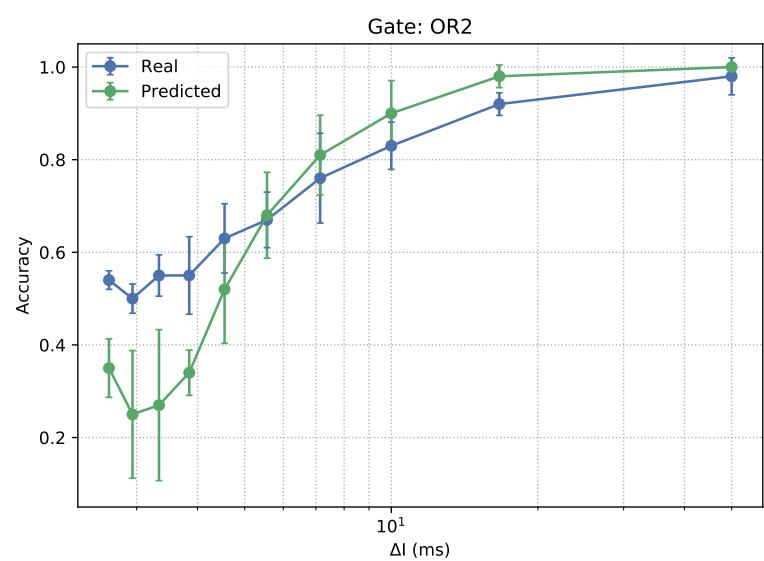

(b)

Fig. 10. Real and predicted mean and standard deviation of the accuracy for an (a) AND gate type 1 and (b) OR gate type 2 in relation to the ISI. Predicted results obtained from the implementation of the model proposed in Section III-B1.

is quite low showing the robustness of developing gates from the various types of neurons. The changes in the curves can be explained as a result of the influence of different types of gates, the variety of morphological types of cells that compose a gate and to the sampling frequency of spike trains during numerical analysis.

\section{B. Epilepsy Case Study}

When simulating a network of cells susceptible to epileptic seizures, the analysis was performed in both cases (with and without logic gates within the network). Only one type of AND gate was used in random positions within the network (as illustrated in Fig. 7) that is composed of L23-MC, L23NBC and L1-HAC cells. The placement of the AND gates was chosen at random and we start placing the gates inside the network at regions with high connections to other cells.

Fig. 12 shows the effect of increasing or decreasing the intensity of an ionic current while the other is kept constant with their initial value. This specific simulation based on the model presented in Section IV was performed with a single Hodgkin-Huxley compartment during $400 \mathrm{~ms}$ while an external current $\left(10 \mu \mathrm{A} / \mathrm{cm}^{2}\right)$ is injected into the compartment in between 100 and $300 \mathrm{~ms}$. The goal of an external stimulus is to evaluate whether there was any spontaneous spike evoked during the simulation. As we can observe, the dynamics between the $K$ and $N a$ influences the spiking rate of the neurons. Based on this, we can replicate the different seizurelike events that are described in Equation (29) with the appropriate ion values.

Figure 13(b) shows how placing a higher number of gates inside the network may help filter out high frequencies of firing by decreasing the average firing rate of the network. This effect is visually shown in Fig. 13(a), where during the seizure, which is possible to generate by manipulating $I_{K}$ and $I_{N a}$ (Fig. 12), the entire network resulted in lower levels of activity with 16 gates in comparison with the network that did not contain any gates.

\section{CONCLUSION}

Even though around 50 million people worldwide suffer from Epilepsy, it is estimated that $10 \%$ of the world population will have a seizure during their lifetime without even being an epileptic person. In this paper, the performance of neuronal logic gates was presented as isolated units and their positive effect by smoothing out the high-frequency firing activity of several brain cells that occur during seizures. This approach requires that the cells involved in the gating process should be synthetically engineered and strategically positioned depending on the network connectivity to improve results.

Part of this work included a proposed model based on queue theory concepts that can predict how accurate a specific gating unit can be based on the input and threshold of the output cell. The model showed, in the worst scenario, for OR gates, an MSE of 0.025 while for AND gates this value is of 0.006 . The results also show that the sampling frequency of the spike train plays a role in the accuracy of the gates and the quality of the model.

Although this paper only concentrated on the treatment of seizures in the brain, logic gates can also be applied for the encoding of information and have the potential to use synthetic biology to create medical bio nano-machines to improve the quality of life of people with neurodegenerative diseases and enhanced information processing inside the brain.

\section{REFERENCES}

[1] T. Nakano, "Molecular communication: A 10 year retrospective," IEEE Transactions on Molecular, Biological and Multi-Scale Communications, vol. 3, no. 2, pp. 71-78, June 2017.

[2] I. F. Akyildiz, M. Pierobon, S. Balasubramaniam, and Y. Koucheryavy, "The internet of bio-nano things," IEEE Communications Magazine, vol. 53, no. 3, pp. 32-40, 2015.

[3] L. Felicetti, M. Femminella, G. Reali, P. Gresele, M. Malvestiti, and J. N. Daigle, "Modeling CD40-Based Molecular Communications in Blood Vessels," IEEE Transactions on NanoBioscience, vol. 13, no. 3, pp. 230-243, Sep. 2014.

[4] L. Felicetti, M. Femminella, G. Reali, and P. Liò, "Applications of molecular communications to medicine: A survey," Nano Communication Networks, vol. 7, pp. 27 - 45, 2016.

[5] E. Balevi and O. B. Akan, "A Physical Channel Model for Nanoscale Neuro-Spike Communications," IEEE Transactions on Communications, vol. 61, no. 3, pp. 1178-1187, March 2013. 


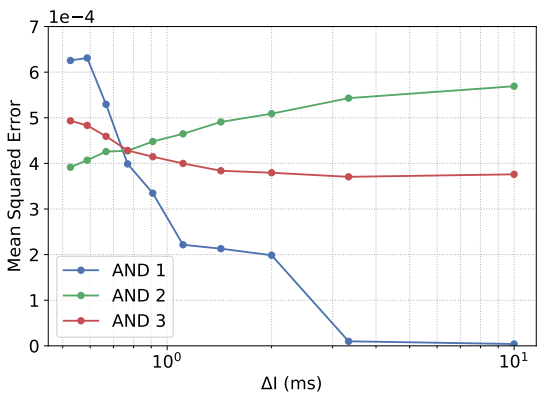

(a) $1 \mathrm{~ms}$.

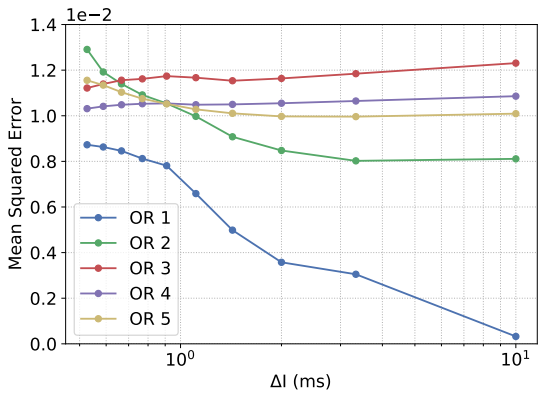

(d) $1 \mathrm{~ms}$.

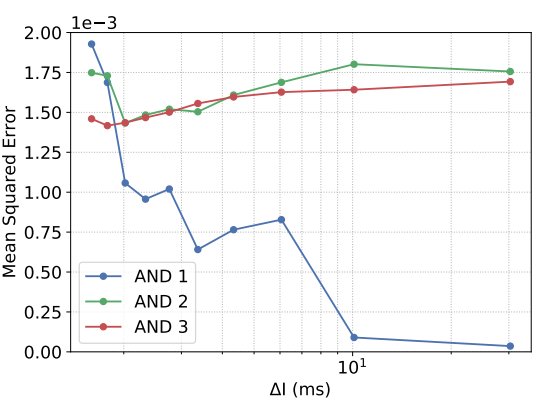

(b) $3 \mathrm{~ms}$.

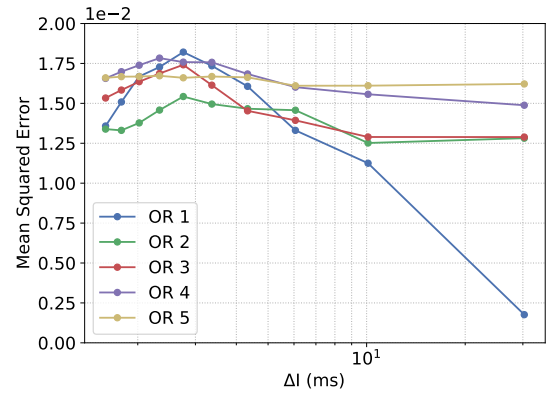

(e) $3 \mathrm{~ms}$

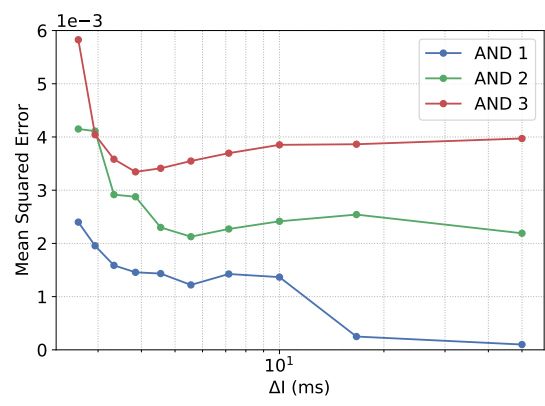

(c) $5 \mathrm{~ms}$.

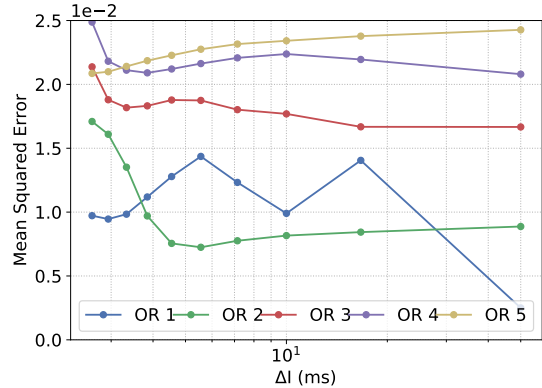

(f) $5 \mathrm{~ms}$.

Fig. 11. Mean Squared Error between the predicted accuracy by the model described in Section III-B1 and accuracy calculated with real output. Spike trains were sampled at different time slots. Figures 11(a), 11(b) and 11(c) show the MSE for all AND gates and Figures 11(d), 11(e) and 11(f) show the MSE for all OR gates.

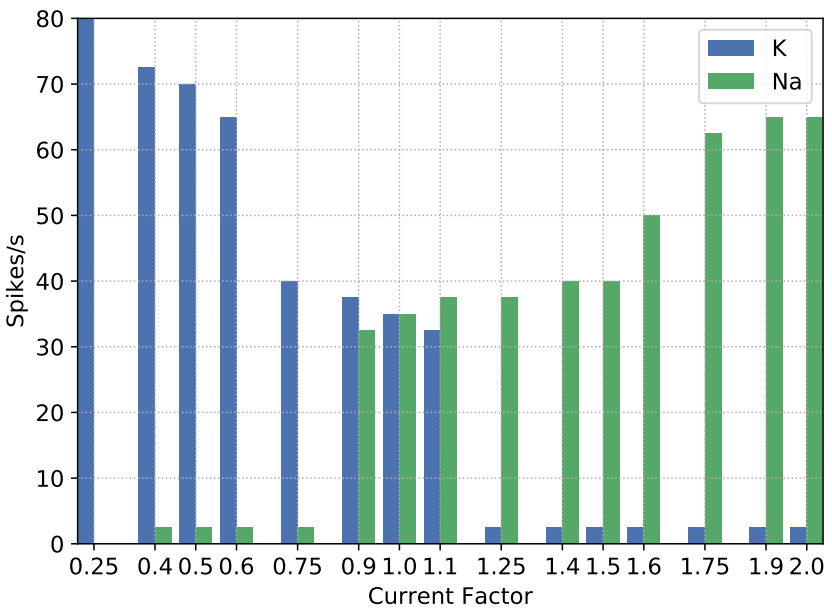

Fig. 12. The effect of multiplying the current factor to the ionic currents When analyzing $I_{K}, I_{N a}$ was kept at their default operation, and vice-versa. It is noticeable how the firing rate increases to values within the range presented in Eq. (29) with the increase of the intensity of ionic currents.

[6] S. Thorpe, A. Delorme, and R. V. Rullen, "Spike-based strategies for rapid processing," Neural Networks, vol. 14, no. 6, pp. 715 - 725, 2001.

[7] F. Zeldenrust, W. J. Wadman, and B. Englitz, "Neural Coding With Bursts - Current State and Future Perspectives," Frontiers in Computational Neuroscience, vol. 12, p. 48, 2018.

[8] G. L. Adonias, M. T. Barros, L. Doyle, and S. Balasubramaniam, "Utilising EEG Signals for Modulating Neural Molecular Communications," in 5th ACM International Conference on Nanoscale Computing and Communication 2018 (ACM NanoCom'18), Reykjavik, Iceland, Sep.
2018.

[9] C. P. Billimoria, R. A. DiCaprio, J. T. Birmingham, L. F. Abbott, and E. Marder, "Neuromodulation of spike-timing precision in sensory neurons," Journal of Neuroscience, vol. 26, no. 22, pp. 5910-5919, 2006.

[10] G.-S. Yi, J. Wang, X.-L. Wei, K.-M. Tsang, W.-L. Chan, and B. Deng, "Neuronal spike initiation modulated by extracellular electric fields," PLOS ONE, vol. 9, no. 5, pp. 1-10, 052014.

[11] J. Choe, B. A. Coffman, D. T. Bergstedt, M. D. Ziegler, and M. E. Phillips, "Transcranial Direct Current Stimulation Modulates Neuronal Activity and Learning in Pilot Training," Frontiers in Human Neuroscience, vol. 10, p. 34, 2016.

[12] C. Agustín-Pavón and M. Isalan, "Synthetic biology and therapeutic strategies for the degenerating brain," BioEssays, vol. 36, no. 10, pp. 979-990, 2014.

[13] W. S. McCulloch and W. Pitts, "A logical calculus of the ideas immanent in nervous activity," The bulletin of mathematical biophysics, vol. 5, no. 4, pp. 115-133, Dec 1943.

[14] A. Goldental, S. Guberman, R. Vardi, and I. Kanter, "A computational paradigm for dynamic logic-gates in neuronal activity," Frontiers in Computational Neuroscience, vol. 8, p. 52, 2014.

[15] J. Larouche and C. A. Aguilar, "New technologies to enhance in vivo reprogramming for regenerative medicine," Trends in biotechnology, 2018.

[16] T. Miyamoto, S. Razavi, R. DeRose, and T. Inoue, "Synthesizing Biomolecule-Based Boolean Logic Gates," ACS Synthetic Biology, vol. 2, no. 2, pp. 72-82, 2013, pMID: 23526588.

[17] T. P. Vogels and L. F. Abbott, "Signal propagation and logic gating in networks of integrate-and-fire neurons," Journal of Neuroscience, vol. 25, no. 46, pp. 10786-10795, 2005.

[18] T. Song, P. Zheng, M. D. Wong, and X. Wang, "Design of logic gates using spiking neural $\mathrm{p}$ systems with homogeneous neurons and astrocytes-like control," Information Sciences, vol. 372, pp. 380 - 391, 2016.

[19] S. A. Benner and A. M. Sismour, "Synthetic biology," Nature Reviews Genetics, vol. 6, no. 7, pp. 533-543, 2005.

[20] M. J. Smanski, H. Zhou, J. Claesen, B. Shen, M. A. Fischbach, and C. A. Voigt, "Synthetic biology to access and expand nature's chemical 

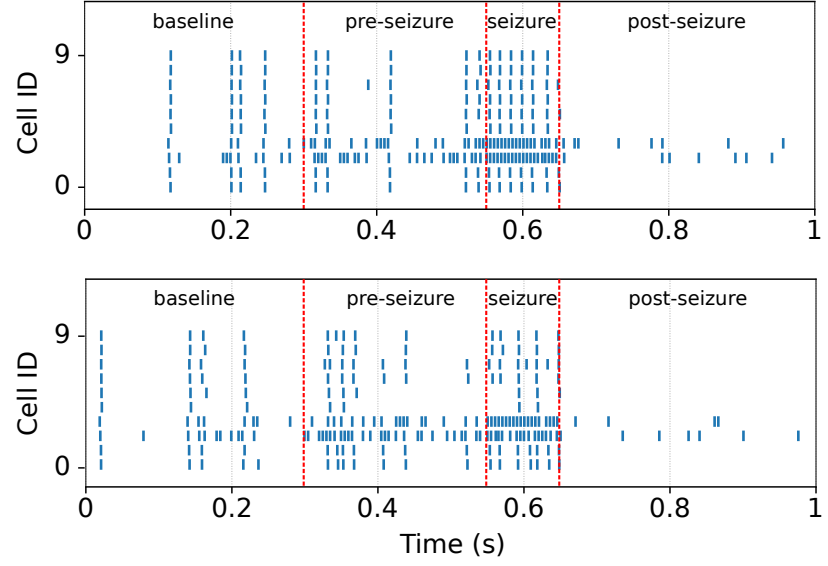

(a) Zero (top) and 16 (bottom) gates in the network.

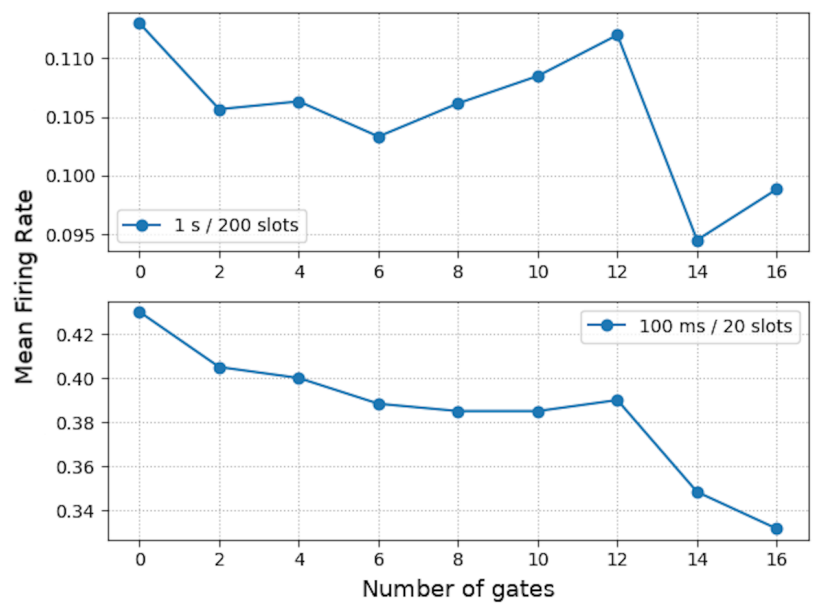

(b) Mean firing rate of the network.

Fig. 13. Simulation of epileptic seizures in a network with 10 neurons ( 2 neurons per cortical layer), stimulation performed in cells at layer $2 / 3$. (a) Raster plot of the network with no gates inserted and natural connections only (top) as illustrated in Fig. 3; and raster plot of the network with 16 neuronal logic gates (bottom), natural connections are broken where gates are placed as depicted in Fig. 7; (b) Mean firing rate in the network as more and more gates are placed within it; top graph shows the firing rate of the whole network for all stages as shown in Fig. 13(a); bottom graph shows the firing rate for the whole network but only for the seizure stage.

diversity," Nature Reviews Microbiology, vol. 14, p. 135, Feb 2016, review Article.

[21] F. Lienert, J. J. Lohmueller, A. Garg, and P. A. Silver, "Synthetic biology in mammalian cells: next generation research tools and therapeutics," Nature Reviews Molecular Cell Biology, vol. 15, no. 2, pp. 95-107, 2014.

[22] I. F. Akyildiz, F. Brunetti, and C. Blázquez, "Nanonetworks: A new communication paradigm," Computer Networks, vol. 52, no. 12, pp. 2260-2279, 2008

[23] G. L. Adonias, A. Yastrebova, M. T. Barros, S. Balasubramaniam, and Y. Koucheryavy, "A Logic Gate Model based on Neuronal Molecular Communication Engineering," in Proceedings of the 4th Workshop on Molecular Communications, Linz, Austria, Apr. 2019.

[24] J. Hasty, D. McMillen, and J. J. Collins, "Engineered gene circuits," Nature, vol. 420, no. 6912, p. 224, 2002

[25] D. Morse and P. Sassone-Corsi, "Time after time: inputs to and outputs from the mammalian circadian oscillators," Trends in neurosciences, vol. 25, no. 12, pp. 632-637, 2002.

[26] R.-S. Wang, A. Saadatpour, and R. Albert, "Boolean modeling in systems biology: an overview of methodology and applications," Physical Biology, vol. 9, no. 5, p. 055001, sep 2012.
[27] W. Hao and A. Friedman, "Mathematical model on Alzheimer's disease," BMC Systems Biology, vol. 10, no. 1, p. 108, 2016.

[28] S. Bakshi, V. Chelliah, C. Chen, and P. H. van der Graaf, "Mathematical Biology Models of Parkinson's Disease," CPT: Pharmacometrics \& Systems Pharmacology, vol. 8, no. 2, pp. 77-86, 2019.

[29] F. Fröhlich, M. Bazhenov, V. Iragui-Madoz, and T. J. Sejnowski, "Potassium dynamics in the epileptic cortex: New insights on an old topic," The Neuroscientist, vol. 14, no. 5, pp. 422-433, 2008, pMID: 18997121.

[30] A. Morano, L. Iannone, C. Palleria, M. Fanella, A. T. Giallonardo, G. De Sarro, E. Russo, and C. Di Bonaventura, "Pharmacology of new and developing intravenous therapies for the management of seizures and epilepsy," Expert opinion on pharmacotherapy, vol. 20, no. 1, pp. 25-39, 2019.

[31] M. C. Li and M. J. Cook, "Deep brain stimulation for drug-resistant epilepsy," Epilepsia, vol. 59, no. 2, pp. 273-290, 2018.

[32] V. K. Jirsa, W. C. Stacey, P. P. Quilichini, A. I. Ivanov, and C. Bernard, "On the nature of seizure dynamics," Brain, vol. 137, no. 8, pp. 22102230, 062014.

[33] Y.-C. Chen et al., "A NeuroD1 AAV-Based Gene Therapy for Functional Brain Repair after Ischemic Injury through In Vivo Astrocyte-to-Neuron Conversion," Molecular Therapy, 2019.

[34] C.-Y. Lin et al., "Non-invasive, neuron-specific gene therapy by focused ultrasound-induced blood-brain barrier opening in Parkinson's disease mouse model," Journal of Controlled Release, vol. 235, pp. $72-81$, 2016.

[35] H. Markram et al., "Reconstruction and Simulation of Neocortical Microcircuitry," Cell, vol. 163, no. 2, pp. 456-492, 2015.

[36] A. Peters, "The morphology of minicolumns," in The neurochemical basis of autism. Springer, 2010, pp. 45-68.

[37] A. Mishra and S. K. Majhi, "A comprehensive survey of recent developments in neuronal communication and computational neuroscience," Journal of Industrial Information Integration, vol. 13, pp. 40 - 54, 2019.

[38] S. Zhou and Y. Yu, "Synaptic E-I Balance Underlies Efficient Neural Coding," Frontiers in Neuroscience, vol. 12, p. 46, 2018.

[39] N. T. Carnevale and M. L. Hines, The NEURON Book, 1st ed. New York, NY, USA: Cambridge University Press, 2009.

[40] L. Long and G. Fang, A Review of Biologically Plausible Neuron Models for Spiking Neural Networks. America Institute of Aeronautics and Astronautics, 2010

[41] M. Pospischil, M. Toledo-Rodriguez, C. Monier, Z. Piwkowska, T. Bal, Y. Frégnac, H. Markram, and A. Destexhe, "Minimal hodgkin-huxley type models for different classes of cortical and thalamic neurons," Biological cybernetics, vol. 99, no. 4-5, pp. 427-441, 2008.

[42] A. L. Hodgkin and A. F. Huxley, "A quantitative description of membrane current and its application to conduction and excitation in nerve," The Journal of physiology, vol. 117, no. 4, pp. 500-544, 1952.

[43] C. Koch, Biophysics of computation: information processing in single neurons. Oxford university press, 2004.

[44] I. F. Akyildiz, M. Pierobon, and S. Balasubramaniam, "An Information Theoretic Framework to Analyze Molecular Communication Systems Based on Statistical Mechanics," Proceedings of the IEEE, vol. 107, no. 7, pp. 1230-1255, July 2019.

[45] J. Platkiewicz and R. Brette, "A Threshold Equation for Action Potential Initiation," PLOS Computational Biology, vol. 6, no. 7, pp. 1-16, 07 2010.

[46] H. Markram, M. Toledo-Rodriguez, Y. Wang, A. Gupta, G. Silberberg, and $\mathrm{C}$. $\mathrm{Wu}$, "Interneurons of the neocortical inhibitory system," Nature Reviews Neuroscience, vol. 5, no. 10, pp. 793-807, 2004.

[47] R. Cowan, "The uncontrolled traffic merge," Journal of Applied Probability, vol. 16, no. 2, p. 384-392, 1979.

[48] E. Toivari, T. Manninen, A. K. Nahata, T. O. Jalonen, and M.-L. Linne, "Effects of transmitters and amyloid-beta peptide on calcium signals in rat cortical astrocytes: Fura-2am measurements and stochastic model simulations," PloS one, vol. 6, no. 3, p. e17914, 2011.

[49] M. T. Barros, W. Silva, and C. D. M. Regis, "The multi-scale impact of the alzheimer's disease on the topology diversity of astrocytes molecular communications nanonetworks," IEEE Access, vol. 6, pp. 78 904-78917, 2018.

[50] D. Reato, M. Cammarota, L. C. Parra, and G. Carmignoto, "Computational model of neuron-astrocyte interactions during focal seizure generation," Frontiers in computational neuroscience, vol. 6, p. 81, 2012.

[51] J. C. Wooley, H. S. Lin, N. R. Council et al., "Computational modeling and simulation as enablers for biological discovery," in Catalyzing inquiry at the interface of computing and biology. National Academies Press (US), 2005. 
[52] E. Barreto and J. R. Cressman, "Ion concentration dynamics as a mechanism for neuronal bursting," Journal of Biological Physics, vol. 37, no. 3, pp. 361-373, Jun 2011.

[53] R. E. Kass and V. Ventura, "A Spike-Train Probability Model," Neural Computation, vol. 13, no. 8, pp. 1713-1720, 2001.

[54] N. Hanisch and M. Pierobon, "Digital modulation and achievable information rates of thru-body haptic communications," in Disruptive Technologies in Sensors and Sensor Systems, vol. 10206. International Society for Optics and Photonics, 2017, p. 1020603.

[55] C. Alvarado-Rojas, K. Lehongre, J. Bagdasaryan, A. Bragin, R. Staba, J. Engel, V. Navarro, and M. LE VAN QUYEN, "Single-unit activities during epileptic discharges in the human hippocampal formation," Frontiers in Computational Neuroscience, vol. 7, p. 140, 2013.

[56] J. Du, V. Vegh, and D. C. Reutens, "Small changes in synaptic gain lead to seizure-like activity in neuronal network at criticality," Scientific Reports, vol. 9, no. 1, p. 1097, 2019.

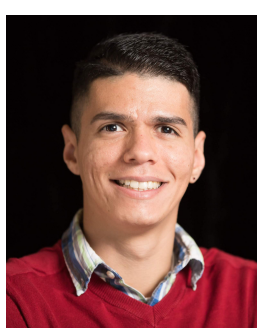

Geoflly L. Adonias is currently a Ph.D. student at the Telecommunications Software \& Systems Group (TSSG), Waterford Institute of Technology (WIT), Ireland, under the Science Foundation Ireland (SFI) CONNECT Project for future networks and communications. He received his B.Eng. in Electrical Engineering from the Federal Institute of Education, Science and Technology of Paraíba (IFPB), Brazil, in 2017. His current research interests include Nanoscale Communications, Digital Signal Processing and Wireless Networks.

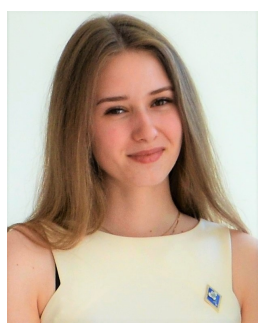

Anastasia Yastrebova has received her B.Sc. in the field of Information Technology and Communication Systems from the Bonch-Bruevich SaintPetersburg State University of Telecommunications, Russia, in 2017 and M.Sc. (dist.) in Information Technology from Tampere University (former Tampere University of Technology), Finland, in 2019. She is currently Research Scientist at the Technical Research Centre of Finland, VTT Ltd. Her research interests include molecular communications, heterogeneous wireless communication networks, and next-generation communication systems.

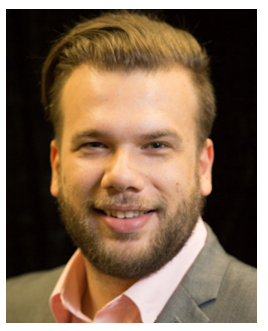

Michael Taynnan Barros is currently the recipient of the Marie Skłodowska-Curie Individual Fellowship (MSCA-IF) at the BioMediTech Institute of the Tampere University, Finland. He received his Ph.D. in Telecommunication Software at the Waterford Institute of Technology, Ireland, in 2016, M.Sc. degree in Computer Science at the Federal University of Campina Grande, Brazil, in 2012 and B.Tech. degree in Telematics at the Federal Institute of Education, Science and Technology of Paraíba, Brazil, in 2011. $\mathrm{He}$ has authored or co-authored over 60 research papers in various international flagship journals and conferences in the areas of wireless communications, molecular and nanoscale communications as well as bionanoscience. He is also a reviewer for many journals and participated as technical program committee and reviewer for various international conferences. Research interests include Internet of BioNanoThings, molecular communications, bionanoscience and $6 \mathrm{G}$ Communications.

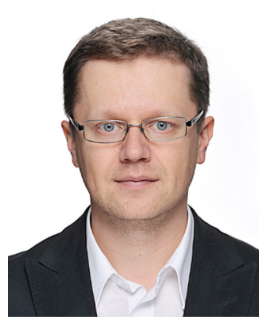

Yevgeni Koucheryavy received his Ph.D. degree from Tampere University of Technology in 2004. $\mathrm{He}$ is a professor with the Unit of Electrical Engineering, Tampere University. His current research interests include various aspects in heterogeneous wireless communication networks and systems, the Internet of Things and its standardization, and nanocommunications. He is an Associate Technical Editor for the IEEE Communications Magazine and an Editor for the IEEE Communications Surveys and Tutorials.

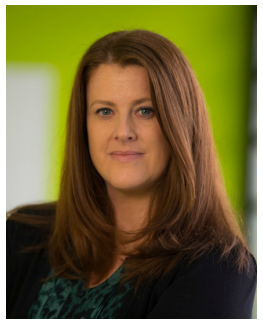

Frances Cleary is a Research Division Manager in TSSG-WIT. Her groups research thematic areas of interest include Bio-Nano Communication, Virtual \& Augmented Reality and Pervasive sensing, Embedded Systems Communications. Frances has worked in multiple research roles from Project Coordinator, Business Development Manager, Technical lead and now as Research Division Manager. The research Division specifically focuses on the application of ICT technologies across the Healthcare and Transport sectors.

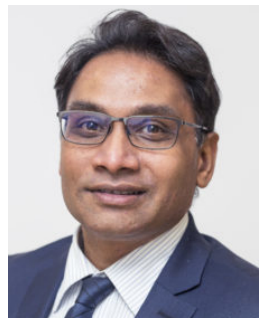

Sasitharan Balasubramaniam (Sasi) received his Bachelors of Engineering (Electrical and Electronic) and $\mathrm{PhD}$ (Computer Science) degrees from the University of Queensland, Australia, in 1998 and 2005 , respectively, and Masters of Engineering Science (Computer and Communication Engineering) degree in 1999 from the Queensland University of Technology, Australia. After completion of his $\mathrm{PhD}$, Sasi joined the Telecommunications Software \& Systems Group (TSSG), Waterford Institute of Technology, Ireland where his research focused on bio-inspired communication networks. In 2009, he successfully received the Science Foundation Ireland Starter Investigator Research Grant, which allowed him to create a Bio-Inspired Research Unit. In 2013, Sasi joined the Department of Electronics and Communication Engineering, Tampere University of Technology, Finland, where in 2014 he received the Academy of Finland Research Fellow grant. As of 2019, Sasi is the Director of Research for the TSSG, and is the Principal Investigator for the recently funded Science Foundation Ireland (SFI) VistaMilk research centre, as well as a Funded Investigator for the SFI CONNECT and SFI FutureNeuro research centres. Sasi has published over 100 journal and conference papers, and actively participates in various conference committees. He is currently the Chair of the Steering Committee for ACM NanoCom, a conference which he co-founded. He is currently an editor for the IEEE Letters of the Computer Society, Elsevier Nano Communication Networks, as well as Elsevier Digital Communication Networks journals. He was a past Associate Editor for the IEEE Internet of Things Journal. In 2018, Sasi was also the IEEE Nanotechnology Council Distinguished Lecturer. His current research interests include molecular and nano communications, terahertz communication for $6 \mathrm{G}$, as well as the Internet of Nano Things. Sasi is currently an IEEE Senior Member. 